\title{
A Robust, Open-Flask, Moisture-Tolerant, and Scalable Route to Unprotected a/ß-Amino Acid N-Carboxyanhydrides
}

\author{
Zi-You Tian, HUA LU
}

Submitted date: 27/12/2020 - Posted date: 29/12/2020

Licence: CC BY-NC-ND 4.0

Citation information: Tian, Zi-You; LU, HUA (2020): A Robust, Open-Flask, Moisture-Tolerant, and Scalable Route to Unprotected a/ß-Amino Acid N-Carboxyanhydrides. ChemRxiv. Preprint. https://doi.org/10.26434/chemrxiv.13489857.v1

Synthetic polypeptides, commonly prepared by the ring-opening polymerization (ROP) of amino acid $\mathrm{N}$-carboxyanhydrides (NCA), are a family of biomimetic materials with vast biomedical applications. A great hurdle in the pro-duction of synthetic polypeptides is the synthesis of NCA, which requires ultra-dry solvents, Schlenk line/gloveboxes, and the protection of sidechain functional groups. Herein, we report a robust and scalable new method for the production of unpro-tected NCA monomers in air and under moisture. The method employs propylene oxide or epichlorohydrin as an inexpensive and ultra-fast scavenger of hydrogen chloride to prevent NCA from acid-catalyzed decomposition under moist conditions. The broad scope and outstanding functional group tolerance of the method are demonstrated by the successful synthesis of more than 30 different NCAs, including many otherwise inaccessible compounds with reactive functional groups (e.g. hy-droxyl, thiol, and carboxylic acid), at high yield and up to ten-gram scale. The scope of the method can be further extended to various a-hydroxyl acid O-carboxyanhydrides (OCA) and $\beta$-amino acid NCAs ( $\beta N C A)$. Given these merits, our strategy holds great potential for revolutionizing the synthesis of NCA and polypeptides, and dramatically expanding the industrial application of synthetic polypeptides

File list (2)

NCA_Chemrxiv_manuscript.docx (6.75 MiB) view on ChemRxiv - download file 


\title{
A Robust, Open-Flask, Moisture-Tolerant, and Scalable Route to Unprotected $\boldsymbol{\alpha} / \boldsymbol{\beta}$-Amino Acid $N$-Carboxyanhydrides
}

\author{
Ziyou Tian, and Hua Lu* \\ Beijing National Laboratory for Molecular Sciences, Center for Soft Matter Science and \\ Engineering, Key Laboratory of Polymer Chemistry and Physics of Ministry of Education, \\ College of Chemistry and Molecular Engineering, Peking University, Beijing 100871, People's \\ Republic of China.
}

\begin{abstract}
Synthetic polypeptides, commonly prepared by the ring-opening polymerization (ROP) of amino acid $\mathrm{N}$-carboxyanhydrides (NCA), are a family of biomimetic materials with vast biomedical applications. A great hurdle in the pro-duction of synthetic polypeptides is the synthesis of NCA, which requires ultra-dry solvents, Schlenk line/gloveboxes, and the protection of side-chain functional groups. Herein, we report a robust and scalable new method for the production of unpro-tected NCA monomers in air and under moisture. The method employs propylene oxide or epichlorohydrin as an inexpensive and ultra-fast scavenger of hydrogen chloride to prevent NCA from acid-catalyzed decomposition under moist conditions. The broad scope and outstanding functional group tolerance of the method are demonstrated by the successful synthesis of more than 30 different NCAs, including many otherwise inaccessible compounds with reactive functional groups (e.g. hy-droxyl, thiol, and carboxylic acid), at high yield and up to ten-gram scale. The scope of the method can be further extended to various $\alpha$ hydroxyl acid $O$-carboxyanhydrides (OCA) and $\beta$-amino acid NCAs ( $\beta N C A)$. Given these merits, our strategy holds great potential for revolutionizing the synthesis of NCA and polypeptides, and dramatically expanding the industrial application of synthetic polypeptides
\end{abstract}

Synthetic polypeptides, commonly prepared by the ring-opening polymerization (ROP) of amino acid $\mathrm{N}$-carboxyanhydrides (NCA), are a family of biomimetic materials that can be applied as catalysts for heterogeneous reactions, self-assembling building blocks, surfactants for double emulsion, drugs carriers, tissue engineering scaffolds, antimicrobial agents, and alternatives to polyethylene glycol (PEG) ${ }^{1-15}$ For example, poly-L-alanine and poly-L-leucine are established industrial catalysts for asymmetric Julia-Colonna epoxidation reactions. ${ }^{16}$ Copaxone ${ }^{\circledR}$, a random co-polypeptide prepared through the ROP of four different NCAs, is a blockbuster drug for multiple sclerosis which generated an annual global sales of 4 billion USD in 2012. ${ }^{17-18}$ Several additional polypeptide-based therapeutic candidates are being tested at different phases of clinical trials. $^{8,19}$ Despite these achievements, synthetic polypeptides are difficult and costly to produce. Although recent advances in ROP reactions has allowed, sometimes even in unpurified solvent or at aqueous-organic interfaces, the rapid generation of polypeptides with predictable and high molar masses $\left(M_{\mathrm{n}}\right)$, low dispersity $(\nexists)$, and well-defined reactive end groups, ${ }^{20-31}$ there are few new methods for simpler synthesis of NCA. The "Leuch's method" for the generation of NCA, which involves the phosgenation and ring-closure of amino acids via (tri)phosgene, is usually carried out under strictly anhydrous and air-free conditions (Scheme 1$).{ }^{32}$ Moreover, traditional workup of NCA is notoriously tricky in that it requires redundant recrystallization in a glovebox using 
expensive dry solvents. Most importantly, although a few functional groups (e.g. alkene, alkyne, azido, ester, halogen, thioether, and selenoether) are tolerated on NCA, ${ }^{33-35}$ the introduction of more nucleophilic and/or proton-bearing functionalities such as alcohol, thiol and carboxylic acid is extremely difficult. Recently, Kramer and Deming achieved the notable breakthrough of purifying non-crystallizable NCAs via silica gel column chromatography in a glovebox. ${ }^{36}$ In another outstanding study, Fuse reported efficient NCA synthesis in a mixture of organic and aqueous solvents with the use of a microflow reactor, followed by a sequence of flash dilution, extraction, and purification. ${ }^{37}$ Despite all these advances, the scalable, open-vessel, and moisturetolerant synthesis of challenging NCAs without the burden of protecting functional side groups remains an urgent and unmet need.

\section{Classic}

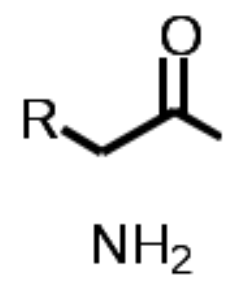

Scheme 1

We began our current study by first seeking to identify the main hurdle in the generation of NCA under regular conditions (i.e. regular solvents without Schlenk line/glove box). The model monomer $\gamma$-benzyl L-glutamate NCA (Bn-GluNCA) was observed to undergo ROP and the reaction reached completion within $4 \mathrm{~h}$ in THF- $d_{8}(2.0 \mathrm{M})$ when stoichiometric $\mathrm{D}_{2} \mathrm{O}$ was added (Figure 1A and S1). The conversion of Bn-GluNCA started out slow but then accelerated, indicating a relatively sluggish initiation by $\mathrm{D}_{2} \mathrm{O}$ compared to the amine-mediated chain propagation. In the presence of both $\mathrm{DCl}$ and $\mathrm{D}_{2} \mathrm{O}$, however, Bn-GluNCA was rapidly hydrolyzed instead of undergoing ROP, as evidenced by the precipitation (Figure 1A and S2). Therefore we concluded that $\mathrm{HCl}$ generated from the synthesis of NCA would cause the latter to rapidly decompose under hydrous conditions. 
A

Figure 1 (A) Decomposition of Bn-GluNCA under different conditions. Inset: NMR tube containing the product of hydrolysized Bn-GluNCA. (B) Rate of HCl-scavenging by PO and $\alpha$-pinene in THF- $d_{8}$ at room temperature. (C) DFT calculation of the ring-closure step in NCA synthesis with or without the assist of PO.

Several studies have demonstrated that the adverse effect of $\mathrm{HCl}$ can be minimized by employing an acid scavenger such as triethylamine (TEA), (+)-limonene, or $\alpha$-pinene, particularly in the synthesis of challenging NCAs. ${ }^{38-40}$ We ruled out the use of TEA because i) it can prematurely initiate the ROP of NCA when in excess, ii) it cannot quench the nucleophilic chloride, which is known to attack the carbonyls of NCAs to form isocyanates, ${ }^{41}$ and iii) the removal of triethylammonium chloride salt is nontrivial. On the other hand, we tested $\alpha$-pinene but failed to improve the product yield or simplify the workup procedures over the conventional method (data not shown). Conducting the model reaction in a mixture of THF- $d_{8}, \alpha$-pinene (1.0 $\mathrm{M})$ and $\mathrm{HCl}(1.0 \mathrm{M})$ for $1 \mathrm{~h}$ at room temperature resulted in low conversion and slow rate (Figure $1 \mathrm{~B}$ and S3). This is not surprising because $\alpha$-pinene has been reported to react with acid at a much slower rate in THF compared to in other solvents such as chloroform and xylene. ${ }^{42}$ Thus, a non- 
basic $\mathrm{HCl}$ scavenger with greater efficiency than $\alpha$-pinene was required.

We hypothesized that an epoxide such as propylene oxide (PO) and epichlorohydrin (ECH) could be ideal additives in NCA synthesis, due to their ability to remove both proton and chloride even at relatively low temperatures (Figure 1B). ${ }^{43}$ To our delight, the use of PO (1.0 M) led to complete, rapid conversion of both reactants in less than $3 \mathrm{~min}$ (Figure 1B) at room temperature, generating a mixture of 1-chloro-2-propanol and 2-chloro-1-propanol (Figure S4). We thus anticipated that PO could greatly facilitate the synthesis of NCA at room temperature, presumably because the acid-catalyzed epoxide ring-opening could generate sufficient heat. Indeed, density functional theory (DFT) calculations confirmed that ethylene oxide could lower the energy barrier of the phosgenation reaction between ${ }_{\mathrm{L}}$-alanine and phosgene by $2.9 \mathrm{kcal} / \mathrm{mol}$ (Figure 1C and S5$6)$.

We then reasoned that the NCA synthesis could be performed under moisture because i) residual water in the solvent could be consumed by the excessive triphosgene, generating $\mathrm{CO}_{2}$ and $\mathrm{HCl}$, ii) all hydrogen chloride could be rapidly removed by PO (Scheme 1), and iii) PO, 1-chloro2-propanol, and 2-chloro-1-propanol are all volatile and easy to remove during the workup. To test

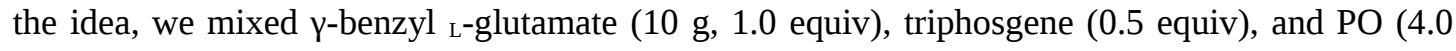
equiv) in regular THF $(150 \mathrm{~mL})$ at room temperature in a sealed $350-\mathrm{mL}$ heavy-wall flask. Unlike the conventional method, which would require heating the reaction mixture to $50-60{ }^{\circ} \mathrm{C}$ for $3-4 \mathrm{~h}$ under nitrogen, our new protocol achieved complete conversion of the amino acid substrate within $\sim 1.5 \mathrm{~h}$ without external heating. Thanks to the efficient and complete elimination of $\mathrm{HCl}$, pure Bn-GluNCA (Scheme 2, 3a) could be obtained at $84 \%$ yield following solvent removal and product recrystallization, under ambient conditions and without the need for anhydrous solvents or a glovebox (environmental humidity: 70\%). The high purity of Bn-GluNCA was confirmed by a combination of ${ }^{1} \mathrm{H}$ and ${ }^{13} \mathrm{C}$ NMR spectroscopy, high-resolution mass spectrometry, and FT-IR spectroscopy (Figure S7). Increasing the loading of PO from 4 to 10 equivalent showed little effect on the yield (See Table S1). The same protocol was then successfully applied to the preparation of $\varepsilon$-carboxybenzyl L-lysine NCA (Z-LysNCA, 3b), $\varepsilon$-trifluoroacetyl L-lysine NCA (TFA-LysNCA, 3c), L-analine NCA (AlaNCA, 3d), L-phenylalanine NCA (PheNCA, 3e), L-valine

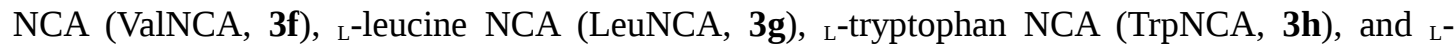
tyrosine NCA (TyrNCA, 3i), all in satisfactory yields (64-90\%) after recrystallization (Scheme 2 and Figure S8-15). However, the yield for glycine NCA (GlyNCA) was below 50\% even when the reaction was extended to $24 \mathrm{~h}$ (See the SI), likely due to the poor solubility of glycine in organic solvents. Surprisingly, complete glycine conversion was achieved within 13 h by simply replacing PO with ECH, affording pure GlyNCA in 76\% yield after recrystallization (Scheme 2, $\mathbf{3} \mathbf{j}$ and Figure S16). While the exact reason of this phenomenon is still unknown, one hypothesis that is under investigation in our lab involves the cooperative hydrogen-halogen bond between glycine and the addition product of ECH, which enhances the solubility of the substrate. Importantly, our method exhibited satisfactory compatibility with normal column chromatography for the synthesis of non-crystallizable NCAs. One notable example was the exceedingly hygroscopic $\gamma$-(2-(2-(2-

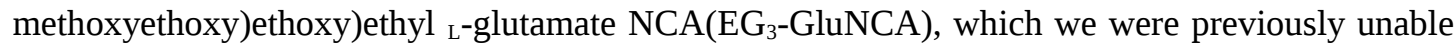
to synthesize at $40 \%$ environmental humidity and above, regardless of how other reaction conditions were optimized. In the current study, however, we successfully obtained $2.7 \mathrm{~g}$ of pure $\mathrm{EG}_{3}$-GluNCA (Scheme 2, 3k and Figure S17) with a separation yield of $72 \%$ by using regular solvents and flash column chromatography under air (environmental humidity: 66). The method 
also proved robust for the synthesis of ${ }_{\mathrm{L}}$-methionine NCA (MetNCA, Scheme 2, 31, Figure S18), O-diethylphospho L-tyrosine NCA (pOEt-TyrNCA, Scheme 2, 3m, Figure S19), O-tert-butyl Lserine NCA ( $t$ Bu-SerNCA, Scheme 2, 3n, Figure S20), and $\varepsilon-N$-Boc ${ }_{\mathrm{L}}$-lysine NCA (Boc-LysNCA, Scheme 2, 3o, Figure S21), the latter two of which contained acid-labile protective groups that were difficult to handle previously. To fully illustrate the water-tolerance of the method, $\mathrm{H}_{2} \mathrm{O}$ (1 euqiv to amino acid) was intentionally added to the reaction mixture, and the synthesis of BnGluNCA still gave satisfactory results by slighlty increasing the loading of triphosegene (to 0.8 euqiv) and $\mathrm{PO}$ (to 6 equiv).

Scheme 2

Pivoting to $N$-substituted NCAs (NNCA), we surprisingly ran into roadblocks in our initial attempts to prepare sarcosine NCA (SarNCA) and ProNCA. Analysis of the crude reaction mixtures revealed the formation of by-products from the aminolysis of PO by the secondary amines (Figure S22) ${ }^{44}$ We thus employed $N$-Boc amino acids as starting materials, which had been previously applied to NCA synthesis with success. To our gratification, we isolated SarNCA in $79 \%$ yield by conducting the reaction in an open flask at $0{ }^{\circ} \mathrm{C}$ to both ensure smooth gas release during the in-situ deprotection of Boc and prevent the loss of PO vapor (Scheme 2, 4a and Figure S23). The same strategy also proved successful in the synthesis of ProNCA (Scheme 2, $\mathbf{4} \mathbf{b}$, and Figure S24) from $N$-Boc L-proline, attaining a purified yield of $72 \%$ even at $74 \%$ environmental humidity.

To further showcase the functional group tolerance of the new method, we explored the generation of challenging NCAs that would otherwise be inaccessible. As reactive anhydrides, NCAs are thought to be incompatible with nucleophiles and/or reactive protons such as hydroxyl, amine, carboxylic acid and thiol. Consistently, the synthesis of hydroxyl-bearing NCAs, such as $\mathrm{L}^{-}$ serine NCA (SerNCA) and ${ }_{\text {L }}$-threonine NCA (ThrNCA), ${ }^{45}$ had been attempted but without 
convincing characterization results. In contrast, we speculated that hydroxy-bearing NCAs should be well tolerated by our method, based on the fact that neither 1-chloro-2-propanol nor 2-chloro-1propanol, the ring-opening products of $\mathrm{PO}$ and $\mathrm{HCl}$, exhibited any detrimental effect on product formation. Indeed, SerNCA (Scheme 3, 3p, and Figure S25) and ThrNCA (Scheme 3, 3q, and Figure S26) could be isolated at $71 \%$ and $46 \%$ yield (environmental humidity $68 \%$ ), respectively. The moderate decrease in the yield of ThrNCA was not due to low substrate conversion but rather the epimerization of the $\alpha$-carbon of threonine during the recrystallization, which led to diastereomers (Figure S27). As a further example, 4-hydroxy s-proline NCA (HypNCA, Scheme 3 , 4c, and Figure S28) was also obtained in $75 \%$ yield by using $N$-Boc 4-hydroxy L-proline as the starting material. As a strong nucleophile, free thiol has not been directly introduced to the side group of NCA before, even though a few papers claimed the generation of such NCAs but without concrete characterization. ${ }^{46}$ To our great surprise, we successfully synthesized pure ${ }_{\text {L-cysteine }}$ NCA (CysNCA, Scheme 3, 3r, Figure S29) bearing a primary thiol, and o-penicillamine NCA (PenNCA, Scheme 3, 3s, Figure S30) with a tertiary thiol. The production of the temperaturesensitive CysNCA was accomplished in $94 \%$ yield via recrystallization at $8-10{ }^{\circ} \mathrm{C}$ (See SI) and could be easily scaled up to $\sim 10$ g per batch (see SI). The unprotected L-glutamic acid NCA (GluNCA, Scheme 3, 3t, Figure S31) was also smoothly prepared with ECH, but not with PO, and $\sim 10$ grams of the product was obtained after recrystallization at a yield of 92\%. Again, it should be emphasized that all syntheses and workup procedures were conducted using regular solvents and without nitrogen protection, and none of these NCAs was found to be attainable using regular method or by adding $\alpha$-pinene as an additive ( $0 \%$ purification yield, Scheme 3 ). Corroborating the synthetic utility of unprotected NCAs, the ROP of GluNCA (3t) smoothly afforded poly- - $^{-}$ glutamic acid (PLG), a therapeutically relevant biodegradable carrier polymer being evaluated in phase 3 clinical trials, in one step with good molecular weight control (Figure S32-33). ${ }^{19}$ This concise synthesis avoided the burdensome deprotection of poly( $\gamma$-benzyl $\mathrm{L}_{\mathrm{L}}$-glutamate) with highly corrosive $\mathrm{HBr}$, leading to substantial time saving and yield increase compared to the conventional two-step procedure of ROP and deprotection (80\% vs. $40 \%$ ). 

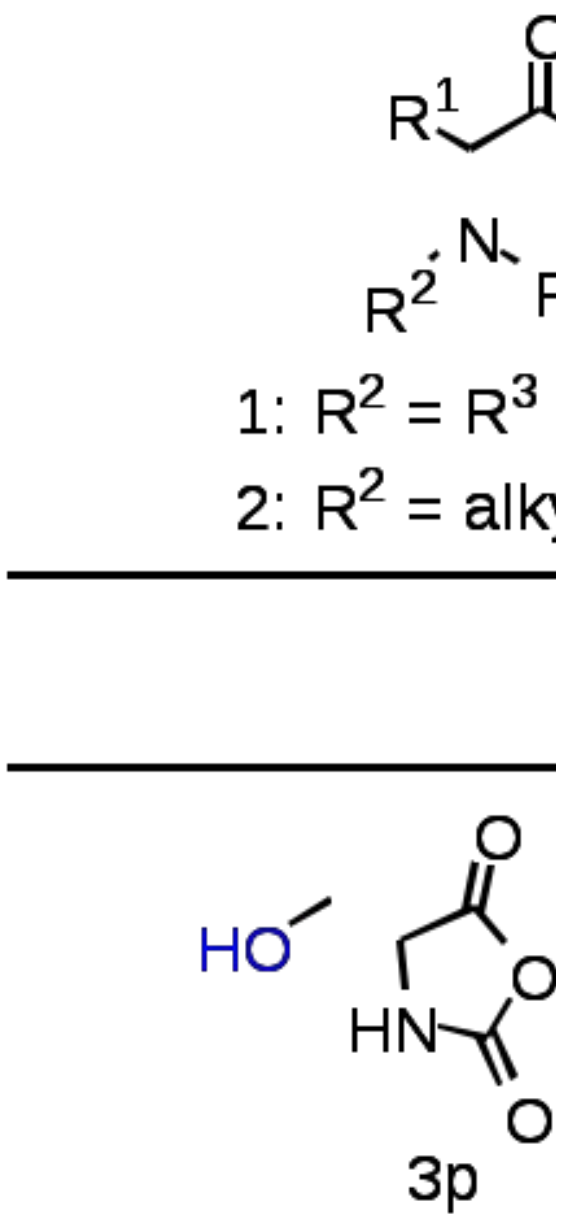

Scheme 3

Building on our success with NCAs, we set out to expand the scope of our new protocol to $\alpha$ hydroxyl acid $O$-carboxyanhydrides (OCA) $)^{47-49}$ and $\beta$-amino acid NCA ( $\left.\beta N C A\right) .{ }^{50}$ Similar to that of NCA, the synthesis of OCA generally involves stringent anhydrous conditions, but also requires activated charcoal to remove impurities. Here, by implementing PO as the acid scavenger, the

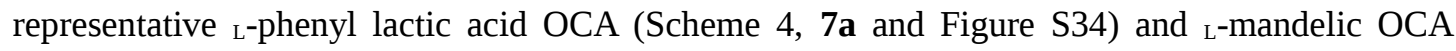
(Scheme 4, 7b and Figure S35) were smoothly produced in modest yields (50-69\%). Of note, the synthesis of OCA required longer time than that of NCA for the reason that the former's hydroxy group is less nucleophilic than the latter's amino group. On the other hand, $\beta$ NCAs, which are considerably more labile than $\alpha$-amino acid NCAs, could also be conveniently synthesized in excellent yields (68-96\%; Scheme 4, 8a-f and Figure S36-41) with ECH as additive and under atmospheric conditions. Notably, $\beta$ NCAs prepared by the flow chemistry method were reported to result in easy product decomposition during the workup or storage. ${ }^{50}$ As a result, only $\mathrm{H}$ NMR yield, but not separation yield, were reported in the study on $\beta$ NCA synthesis. Here, the enhanced stability of $\beta$ NCA suggested an absence of side reactions due to the complete removal of $\mathrm{HCl}$, which once again underscored the power of our method. 
Synthetic polypeptides have gained popularity as biodegradable and protein-mimicking polymers with broad application potential, but the preparation of the NCA monomers requires stringent moisture-free conditions and tricky workup procedures. Acid scavengers, such as TEA and $\alpha$-pinene, have been tested in an attempt to minimize the adverse effect of moisture and improve functional group compatibility, but with limited successes. Furthermore, the detrimental role of $\mathrm{HCl}$ in the formation of NCA, long recognized in the field, has not been elucidated from a mechanistic perspective until this work. After ruling out TEA and $\alpha$-pinene, we identified PO and $\mathrm{ECH}$ as ultra-fast and clean $\mathrm{HCl}$-quenching reagents. We boldly proposed and later successfully proved, for the first time, that the above epoxides could be used to efficiently synthesize NCA under mild, atmospheric conditions, without the need for moisture removal. Subsequent investigations showed that our method could be generally applied to a broad scope of substrates, and could even be executed on a decagram scale to produce challenging NCAs that carry highly reactive $-\mathrm{OH},-\mathrm{COOH}$, and $-\mathrm{SH}$ groups. The use of unprotected NCAs, such as HypNCA, SerNCA, and GluNCA, can undoubtedly improve the efficiency of polypeptide synthesis by obviating the need for tedious protection and deprotection. This is vividly demonstrated through the direct ROP of GluNCA (3t). Moreover, CysNCA could be treated as an inimer, allowing onepot production of hyperbranched polypeptides. The generality of the method was further demonstrated by the synthesis of several OCAs and $\beta$ NCAs in high purity, paving the road for easier access to various functional poly( $\alpha$-hydroxyl acid)s and $\beta$-polypeptides. These works will be reported in separated papers shortly. Last but not least, we expect the method detailed in this study to be readily adaptable to industrial-scale production for reasons that i) both $\mathrm{PO}$ and $\mathrm{ECH}$ are extremely inexpensive and easily available, ii) the reactions can be performed without the need for ultra-dry solvents, nitrogen protection or glovebox, leading to much simpler procedures and considerable cost reduction; iii) the instant removal of $\mathrm{HCl}$ can prevent the erosion of equipment, and iv) the solvents, $\mathrm{PO}, \mathrm{ECH}$, and the ring-opening byproducts can all be easily recycled through 
distillation. Taken together, the simplicity, robustness and scalability of our method promises may ultimately revolutionize the NCA synthesis, profoundly facilitate the popularization of synthetic polypeptides in both laboratories and industry, and open up numerous possibilities for the development of novel, high-performance polypeptide materials.

\section{Supporting Information}

Experimental details, ${ }^{1} \mathrm{H}$ and ${ }^{13} \mathrm{C}$ NMR spectra, kinetic studies, DFT calculations.

$\mathrm{X}$-ray crystallographic data (3p)

X-ray crystallographic data (3q)

X-ray crystallographic data (3r)

\section{Notes}

A provisional patent application has been filed by H.L. and Z.Y.T.

\section{Corresponding Author}

*chemhualu@pku.edu.cn.

\section{Acknowledgment}

This work is supported by the National Natural Science Foundation of China (21722401 and 21975004) and Li Ge-Zhao Ning Life Science Research Foundation for Young Scholars. Z. Y. T. was supported by the National Postdoctoral Program for Innovative Talents of China (BX20190004). The computation was supported by High-performance Computing Platform of Peking University. The authors thank Mr. Shuo Wang for his help on the recrystallization of CysNCA.

\section{References}

1. Rasines Mazo, A.; Allison-Logan, S.; Karimi, F.; Chan, N. J.; Qiu, W.; Duan, W.; O'BrienSimpson, N. M.; Qiao, G. G., Ring opening polymerization of alpha-amino acids: advances in synthesis, architecture and applications of polypeptides and their hybrids. Chem. Soc. Rev. 2020, 49 (14), 4737-4834.

2. Shen, Y.; Fu, X.; Fu, W.; Li, Z., Biodegradable stimuli-responsive polypeptide materials prepared by ring opening polymerization. Chem. Soc. Rev. 2015, 44 (3), 612-22.

3. Hadjichristidis, N.; Iatrou, H.; Pitsikalis, M.; Sakellariou, G., Synthesis of Well-Defined Polypeptide-Based Materials via the Ring-Opening Polymerization of alpha-Amino Acid NCarboxyanhydrides. Chem. Rev. 2009, 109 (11), 5528-5578.

4. Klok, H. A.; Lecommandoux, S., Solid-state structure, organization and properties of peptidesynthetic hybrid block copolymers. Adv. Polym. Sci. 2006, 202, 75-111.

5. Hou, Y.; Zhou, Y.; Wang, H.; Sun, J.; Wang, R.; Sheng, K.; Yuan, J.; Hu, Y.; Chao, Y.; Liu, Z.; Lu, H., Therapeutic Protein PEPylation: The Helix of Nonfouling Synthetic Polypeptides Minimizes Antidrug Antibody Generation. ACS Cent. Sci. 2019, 5 (2), 229-236.

6. Hou, Y.; Lu, H., Protein PEPylation: A New Paradigm of Protein-Polymer Conjugation. Bioconjugate Chem. 2019, 30 (6), 1604-1616.

7. Zagorodko, O.; Arroyo-Crespo, J. J.; Nebot, V. J.; Vicent, M. J., Polypeptide-Based Conjugates as Therapeutics: Opportunities and Challenges. Macromol. Biosci. 2017, 17 (1), 1600316. 
8. Cabral, H.; Kataoka, K., Progress of drug-loaded polymeric micelles into clinical studies. $J$. Controlled Release 2014, 190, 465-476.

9. Anderson, M. A.; O'Shea, T. M.; Burda, J. E.; Ao, Y.; Barlatey, S. L.; Bernstein, A. M.; Kim, J. H.; James, N. D.; Rogers, A.; Kato, B.; Wollenberg, A. L.; Kawaguchi, R.; Coppola, G.; Wang, C.; Deming, T. J.; He, Z. G.; Courtine, G.; Sofroniew, M. V., Required growth facilitators propel axon regeneration across complete spinal cord injury. Nature 2018, 561 (7723), 396-400.

10. Sun, Y. T.; Wollenberg, A. L.; O'Shea, T. M.; Cui, Y. X.; Zhou, Z. H.; Sofroniew, M. V.; Deming, T. J., Conformation-Directed Formation of Self-Healing Diblock Copolypeptide Hydrogels via Polyion Complexation. J. Am. Chem. Soc. 2017, 139 (42), 15114-15121.

11. Xiong, M.; Lee, M. W.; Mansbach, R. A.; Song, Z.; Bao, Y.; Peek, R. M.; Yao, C.; Chen, L. F.; Ferguson, A. L.; Wong, G. C. L.; Cheng, J. J., Helical antimicrobial polypeptides with radial amphiphilicity. Proc. Natl. Acad. Sci. U. S. A. 2015, 112 (43), 13155-13160.

12. Lam, S. J.; O'Brien-Simpson, N. M.; Pantarat, N.; Sulistio, A.; Wong, E. H. H.; Chen, Y. Y.; Lenzo, J. C.; Holden, J. A.; Blencowe, A.; Reynolds, E. C.; Qiao, G. G., Combating multidrugresistant Gram-negative bacteria with structurally nanoengineered antimicrobial peptide polymers. Nat. Microbiol. 2016, 1 (11), 16162.

13. Hanson, J. A.; Chang, C. B.; Graves, S. M.; Li, Z. B.; Mason, T. G.; Deming, T. J., Nanoscale double emulsions stabilized by single-component block copolypeptides. Nature 2008, 455 (7209), 85-88.

14. Song, Z.; Han, Z.; Lv, S.; Chen, C.; Chen, L.; Yin, L.; Cheng, J., Synthetic polypeptides: from polymer design to supramolecular assembly and biomedical application. Chem. Soc. Rev. 2017, 46 (21), 6570-6599.

15. Manai, G.; Houimel, H.; Rigoulet, M.; Gillet, A.; Fazzini, P.-F.; Ibarra, A.; Balor, S.; Roblin, P.; Esvan, J.; Coppel, Y.; Chaudret, B.; Bonduelle, C.; Tricard, S., Bidimensional lamellar assembly by coordination of peptidic homopolymers to platinum nanoparticles. Nat. Commun. 2020, 11 (1), 2051.

16. Baars, S.; Drauz, K. H.; Krimmer, H. P.; Roberts, S. M.; Sander, J.; Skidmore, J.; Zanardi, G., Development of the Julia-Colonna asymmetric epoxidation reaction: Part 1. Preparation and activation of the polyleucine catalyst. Org. Process Res. Dev. 2003, 7 (4), 509-513.

17. Racke, M. K.; Lovett-Racke, A. E.; Karandikar, N. J., The mechanism of action of glatiramer acetate treatment in multiple sclerosis. Neurology 2010, 74 (1), S25-S30.

18. Song, Z. Y.; Khaw, Y. M.; Pacheco, L. A.; Tseng, K. Y.; Tan, Z. Z.; Cai, K. M.; Ponnusamy, E.; Cheng, J. J.; Inoue, M., Induction of a higher-ordered architecture in glatiramer acetate improves its biological efficiency in an animal model of multiple sclerosis. Biomater. Sci. 2020, 8 (19), 5271-5281.

19. Zhao, J.; Koay, E. J.; Li, T.; Wen, X.; Li, C., A hindsight reflection on the clinical studies of poly(l-glutamic acid)-paclitaxel. Wiley Interdiscip. Rev. Nanomed. Nanobiotechnol. 2018, 10 (3), e1497.

20. Zhao, W.; Lv, Y.; Li, J.; Feng, Z.; Ni, Y.; Hadjichristidis, N., Fast and selective organocatalytic ring-opening polymerization by fluorinated alcohol without a cocatalyst. Nat. Commun. 2019, 10 (1), 3590.

21. Grazon, C.; Salas-Ambrosio, P.; Ibarboure, E.; Buol, A.; Garanger, E.; Grinstaff, M. W.; Lecommandoux, S.; Bonduelle, C., Aqueous Ring-Opening Polymerization-Induced SelfAssembly (ROPISA) of N-Carboxyanhydrides. Angew. Chem., Int. Ed. 2020, 59 (2), 622-626. 
22. Song, Z.; Fu, H.; Wang, J.; Hui, J.; Xue, T.; Pacheco, L. A.; Yan, H.; Baumgartner, R.; Wang, Z.; Xia, Y.; Wang, X.; Yin, L.; Chen, C.; Rodríguez-López, J.; Ferguson, A. L.; Lin, Y.; Cheng, J., Synthesis of polypeptides via bioinspired polymerization of in situ purified $\mathrm{N}$-carboxyanhydrides. Proc. Natl. Acad. Sci. U. S. A. 2019, 116 (22), 10658-10663.

23. Wu, Y.; Zhang, D.; Ma, P.; Zhou, R.; Hua, L.; Liu, R., Lithium hexamethyldisilazide initiated superfast ring opening polymerization of alpha-amino acid N-carboxyanhydrides. Nat. Commun. 2018, 9 (1), 5297.

24. Yuan, J.; Zhang, Y.; Li, Z.; Wang, Y.; Lu, H., A S-Sn Lewis Pair-Mediated Ring-Opening Polymerization of $\alpha$-Amino Acid N-Carboxyanhydrides: Fast Kinetics, High Molecular Weight, and Facile Bioconjugation. ACS Macro Lett. 2018, 7 (8), 892-897.

25. Yuan, J.; Sun, Y.; Wang, J.; Lu, H., Phenyl Trimethylsilyl Sulfide-Mediated Controlled RingOpening Polymerization of alpha-Amino Acid N-Carboxyanhydrides. Biomacromolecules 2016, 17 (3), 891-896.

26. Jacobs, J.; Pavlović, D.; Prydderch, H.; Moradi, M.-A.; Ibarboure, E.; Heuts, J. P. A.; Lecommandoux, S.; Heise, A., Polypeptide Nano particles Obtained from Emulsion Polymerization of Amino Acid N-Carboxyanhydrides. J. Am. Chem. Soc. 2019, 141 (32), 1252212526.

27. Cao, J. B.; Siefker, D.; Chan, B. A.; Yu, T. Y.; Lu, L.; Saputra, M. A.; Fronczek, F. R.; Xie, W. W.; Zhang, D. H., Interfacial Ring-Opening Polymerization of Amino-Acid-Derived NThiocarboxyanhydrides Toward Well-Defined Polypeptides. ACS Macro Lett. 2017, 6 (8), 836840.

28. Tao, X. F.; Li, M. H.; Ling, J., alpha-Amino acid N-thiocarboxyanhydrides: A novel synthetic approach toward poly(alpha-amino acid)s. Eur. Polym. J. 2018, 109, $26-42$.

29. Conejos-Sanchez, I.; Duro-Castano, A.; Birke, A.; Barz, M.; Vicent, M. J., A controlled and versatile NCA polymerization method for the synthesis of polypeptides. Polym. Chem. 2013, 4 (11), 3182-3186.

30. Zou, J.; Fan, J. W.; He, X.; Zhang, S. Y.; Wang, H.; Wooley, K. L., A Facile Glovebox-Free Strategy To Significantly Accelerate the Syntheses of Well-Defined Polypeptides by NCarboxyanhydride (NCA) Ring-Opening Polymerizations. Macromolecules 2013, 46 (10), 42234226.

31. Huesmann, D.; Klinker, K.; Barz, M., Orthogonally reactive amino acids and end groups in NCA polymerization. Polym. Chem. 2017, 8 (6), 957-971.

32. Kricheldorf, H. R., Polypeptides and 100 years of chemistry of alpha-amino acid Ncarboxyanhydrides. Angew. Chem., Int. Ed. 2006, 45 (35), 5752-5784.

33. Deming, T. J., Synthesis of Side-Chain Modified Polypeptides. Chem. Rev. 2016, 116 (3), 786808.

34. Song, Z. Y.; Tan, Z. Z.; Cheng, J. J., Recent Advances and Future Perspectives of Synthetic Polypeptides from N-Carboxyanhydrides. Macromolecules 2019, 52 (22), 8521-8539.

35. Wu, G. Q.; Ge, C. L.; Liu, X. Y.; Wang, S.; Wang, L. T.; Yin, L. C.; Lu, H., Synthesis of water soluble and multi-responsive selenopolypeptides via ring-opening polymerization of $\mathrm{N}$ carboxyanhydrides. Chem. Commum. 2019, 55 (54), 7860-7863.

36. Kramer, J. R.; Deming, T. J., General Method for Purification of alpha-Amino acid-Ncarboxyanhydrides Using Flash Chromatography. Biomacromolecules 2010, 11 (12), 3668-3672.

37. Otake, Y.; Nakamura, H.; Fuse, S., Rapid and Mild Synthesis of Amino Acid N-Carboxy 
Anhydrides: Basic-to-Acidic Flash Switching in a Microflow Reactor. Angew. Chem., Int. Ed. 2018, 57 (35), 11389-11393.

38. Fabrice Cornille, B. S. Y.; Jean-Luc Copier, A.; Jean-Pierre Senet, B.; Yves Robin, V. L. P. PROCESS FOR THE PREPARATION OF N-CARBOXYANHYDRIDES. US 6,479,665 B2, 2002.

39. Iizuka, Y.; Uchida, C.; Wakamatsu, K.; Oya, M., Synthesis and Properties of High-MolecularWeight Polypeptides Containing Proline. Bull. Chem. Soc. Jpn. 1993, 66 (4), 1269-1272.

40. Smeets, N. M. B.; van der Weide, P. L. J.; Meuldijk, J.; Vekemans, J. A. J. M.; Hulshof, L. A., A scalable synthesis of L-leucine-N-carboxyanhydride. Org. Process Res. Dev. 2005, 9 (6), 757763.

41. Iwakura, Y.; Uno, K.; Kang, S., The Synthesis and Reactions of 2-Isocyanatoacyl Chlorides. J. Org. Chem. 1965, 30 (4), 1158-1161.

42. Hennion, G. F.; Irwin, C. F., Solvent Effects in Addition Reactions. II. Addition of Hydrogen Bromide and Chloride to $\alpha$-Pinene. J. Am. Chem. Soc. 1941, 63 (3), 860-862.

43. Stewart, C. A.; Vanderwerf, C. A., Reaction of Propylene Oxide with Hydrogen Halides. $J$. Am. Chem. Soc. 1954, 76 (5), 1259-1264.

44. Lehmann, J., Lactone, 2. Mitt.1) Synthese dihydroxylierter Diphenylalkylamine über Azalactone. Archiv. der Pharmazie. 1983, 316 (4), 339-346.

45. Saito, T., A New Rearrangement Reaction of Beta-Hydroxy-Alpha-Amino Acid N-Carboxy Anhydrides to 2-Oxazolidone Derivatives. Bull. Chem. Soc. Jpn. 1964, 37 (5), 624-628.

46. Hirschmann, R.; Schwam, H.; Strachan, R. G.; Schoenewaldt, E. F.; Barkemeyer, H.; Miller, S. M.; Conn, J. B.; Garsky, V.; Veber, D. F.; Denkewalter, R. G., Controlled synthesis of peptides in aqueous medium. VIII. Preparation and use of novel .alpha.-amino acid N-carboxyanhydrides. $J$. Am. Chem. Soc. 1971, 93 (11), 2746-2754.

47. du Boullay, O. T.; Marchal, E.; Martin-Vaca, B.; Cossio, F. P.; Bourissou, D., An activated equivalent of lactide toward organocatalytic ring-opening polymerization. J. Am. Chem. Soc. 2006, 128 (51), 16442-16443.

48. Wang, R. B.; Zhang, J. W.; Yin, Q.; Xu, Y. X.; Cheng, J. J.; Tong, R., Controlled Ring-Opening Polymerization of O-Carboxyanhydrides Using a beta-Diiminate Zinc Catalyst. Angew. Chem., Int. Ed. 2016, 55 (42), 13010-13014.

49. Li, M. S.; Tao, Y.; Tang, J. D.; Wang, Y. C.; Zhang, X. Y.; Tao, Y. H.; Wang, X. H., Synergetic Organocatalysis for Eliminating Epimerization in Ring-Opening Polymerizations Enables Synthesis of Stereoregular Isotactic Polyester. J. Am. Chem. Soc. 2019, 141 (1), 281-289.

50. Sugisawa, N.; Otake, Y.; Nakamura, H.; Fuse, S., Single-Step, Rapid, and Mild Synthesis of beta-Amino Acid N-Carboxy Anhydrides Using Micro-Flow Technology. Chem-Asian J. 2020, 15 (1), 79-84. 


\title{
A Robust, Open-Flask, Moisture-Tolerant, and Scalable Route to Unprotected $\alpha / \beta$ - Amino Acid $N$-Carboxyanhydrides
}

\author{
Ziyou Tian, and Hua Lu* \\ Beijing National Laboratory for Molecular Sciences, Center for Soft Matter Science and \\ Engineering, Key Laboratory of Polymer Chemistry and Physics of Ministry of Education, College \\ of Chemistry and Molecular Engineering, Peking University, Beijing 100871, People's Republic of \\ China.
}

\begin{abstract}
Synthetic polypeptides, commonly prepared by the ring-opening polymerization (ROP) of amino acid $\mathrm{N}$-carboxyanhydrides (NCA), are a family of biomimetic materials with vast biomedical applications. A great hurdle in the pro-duction of synthetic polypeptides is the synthesis of NCA, which requires ultra-dry solvents, Schlenk line/gloveboxes, and the protection of sidechain functional groups. Herein, we report a robust and scalable new method for the production of unpro-tected NCA monomers in air and under moisture. The method employs propylene oxide or epichlorohydrin as an inexpensive and ultra-fast scavenger of hydrogen chloride to prevent NCA from acid-catalyzed decomposition under moist conditions. The broad scope and outstanding functional group tolerance of the method are demonstrated by the successful synthesis of more than 30 different NCAs, including many otherwise inaccessible compounds with reactive functional groups (e.g. hy-droxyl, thiol, and carboxylic acid), at high yield and up to ten-gram scale. The scope of the method can be further extended to various $\alpha$-hydroxyl acid $O$-carboxyanhydrides (OCA) and $\beta$-amino acid NCAs ( $\beta \mathrm{NCA}$ ). Given these merits, our strategy holds great potential for revolutionizing the synthesis of NCA and polypeptides, and dramatically expanding the industrial application of synthetic polypeptides
\end{abstract}

Synthetic polypeptides, commonly prepared by the ring-opening polymerization (ROP) of amino acid $N$-carboxyanhydrides (NCA), are a family of biomimetic materials that can be applied as catalysts for heterogeneous reactions, self-assembling building blocks, surfactants for double emulsion, drugs carriers, tissue engineering scaffolds, antimicrobial agents, and alternatives to polyethylene glycol (PEG). ${ }^{1-15}$ For example, poly-L-alanine and poly-L-leucine are established industrial catalysts for asymmetric Julia-Colonna epoxidation reactions ${ }^{16}{ }^{1}$ Copaxone ${ }^{\circledR}$, a random copolypeptide prepared through the ROP of four different NCAs, is a blockbuster drug for multiple sclerosis which generated an annual global sales of 4 billion USD in 2012. ${ }^{17-18}$ Several additional polypeptide-based therapeutic candidates are being tested at different phases of clinical trials. ${ }^{8}, 19$ Despite these achievements, synthetic polypeptides are difficult and costly to produce. Although recent advances in ROP reactions has allowed, sometimes even in unpurified solvent or at aqueousorganic interfaces, the rapid generation of polypeptides with predictable and high molar masses $\left(M_{\mathrm{n}}\right)$, low dispersity $(\nexists)$, and well-defined reactive end groups, ${ }^{20-31}$ there are few new methods for simpler synthesis of NCA. The "Leuch's method" for the generation of NCA, which involves the phosgenation and ring-closure of amino acids via (tri)phosgene, is usually carried out under strictly anhydrous and air-free conditions (Scheme 1). ${ }^{32}$ Moreover, traditional workup of NCA is notoriously tricky in that it requires redundant recrystallization in a glovebox using expensive dry 
solvents. Most importantly, although a few functional groups (e.g. alkene, alkyne, azido, ester, halogen, thioether, and selenoether) are tolerated on $\mathrm{NCA},{ }^{33-35}$ the introduction of more nucleophilic and/or proton-bearing functionalities such as alcohol, thiol and carboxylic acid is extremely difficult. Recently, Kramer and Deming achieved the notable breakthrough of purifying non-crystallizable NCAs via silica gel column chromatography in a glovebox. ${ }^{36}$ In another outstanding study, Fuse reported efficient NCA synthesis in a mixture of organic and aqueous solvents with the use of a microflow reactor, followed by a sequence of flash dilution, extraction, and purification. ${ }^{37}$ Despite all these advances, the scalable, open-vessel, and moisture-tolerant synthesis of challenging NCAs without the burden of protecting functional side groups remains an urgent and unmet need.

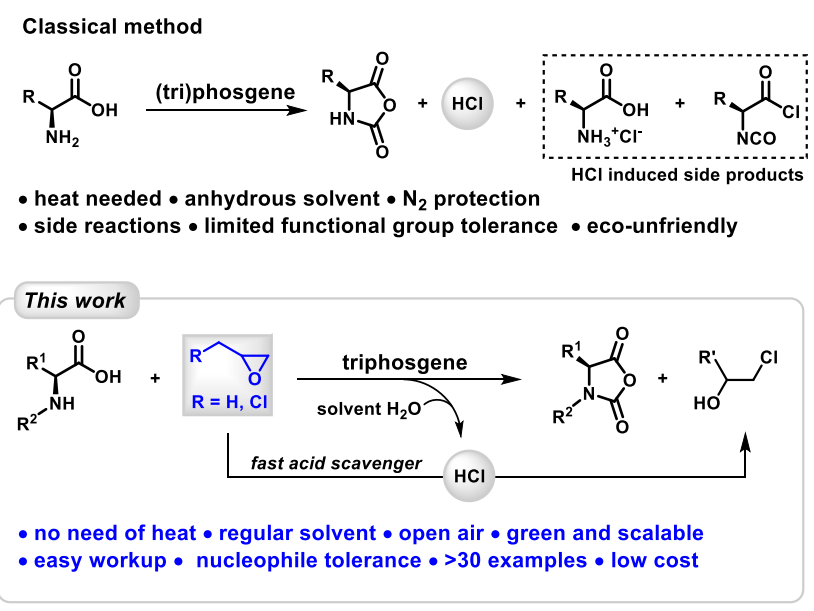

Scheme 1

We began our current study by first seeking to identify the main hurdle in the generation of NCA under regular conditions (i.e. regular solvents without Schlenk line/glove box). The model

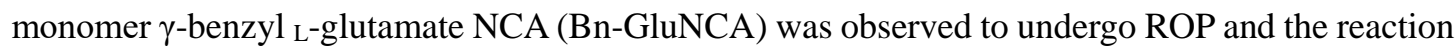
reached completion within $4 \mathrm{~h}$ in THF- $d_{8}(2.0 \mathrm{M})$ when stoichiometric $\mathrm{D}_{2} \mathrm{O}$ was added (Figure $1 \mathrm{~A}$ and S1). The conversion of Bn-GluNCA started out slow but then accelerated, indicating a relatively sluggish initiation by $\mathrm{D}_{2} \mathrm{O}$ compared to the amine-mediated chain propagation. In the presence of both $\mathrm{DCl}$ and $\mathrm{D}_{2} \mathrm{O}$, however, Bn-GluNCA was rapidly hydrolyzed instead of undergoing ROP, as evidenced by the precipitation (Figure $1 \mathrm{~A}$ and $\mathrm{S} 2$ ). Therefore we concluded that $\mathrm{HCl}$ generated from the synthesis of NCA would cause the latter to rapidly decompose under hydrous conditions. 
A

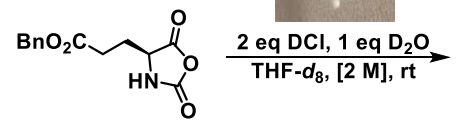

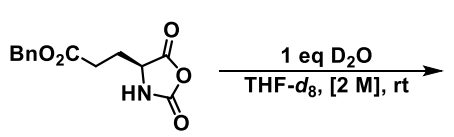

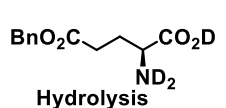

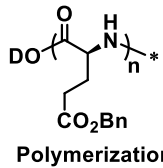
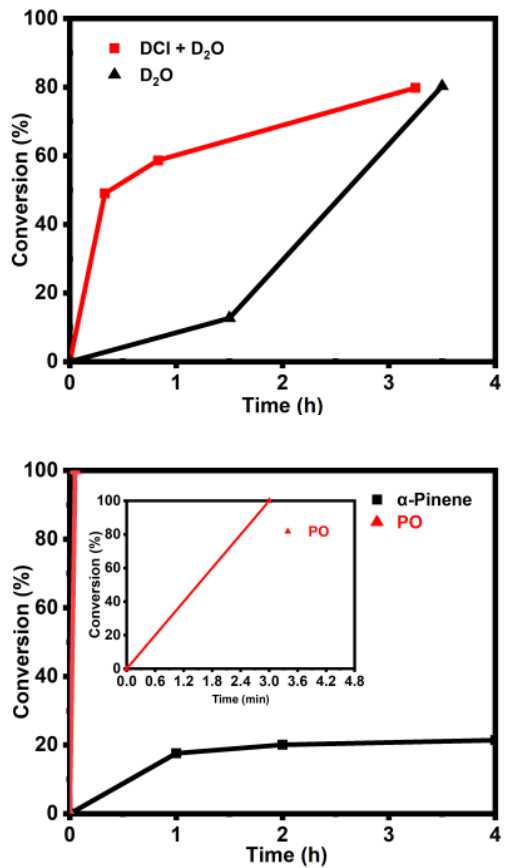

C $\quad \uparrow \begin{aligned} & {[\Delta G]_{\text {sol }}} \\ & \text { in } \mathrm{kcal} / \mathrm{mo}\end{aligned}$

B
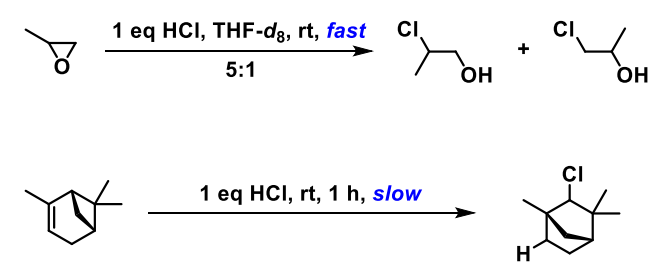

M06-2X/def2-TZVPP//B3LYP/6-31G(d)

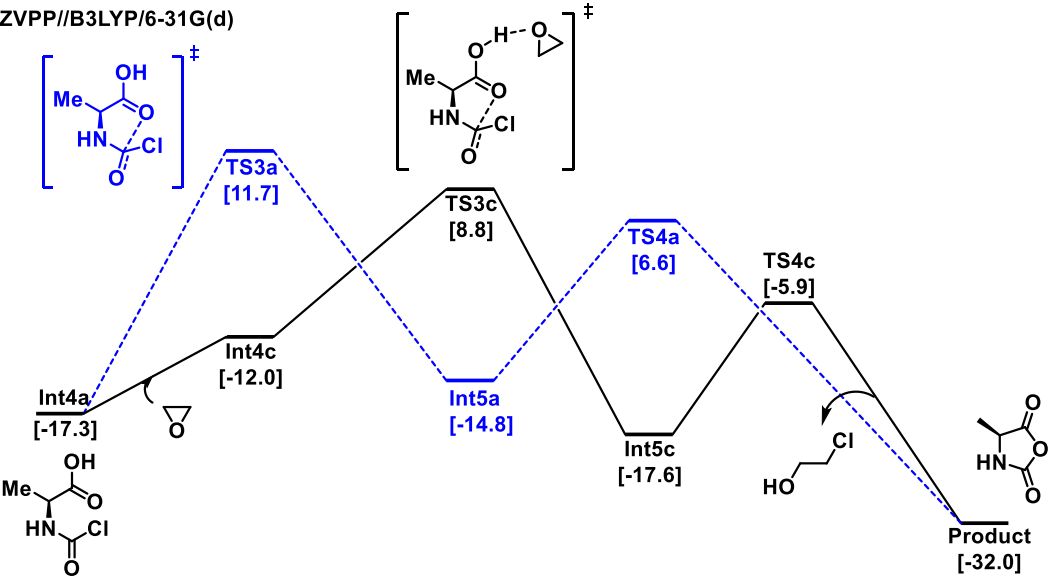

Figure 1 (A) Decomposition of Bn-GluNCA under different conditions. Inset: NMR tube containing the product of hydrolysized Bn-GluNCA. (B) Rate of HCl-scavenging by PO and $\alpha$-pinene in THF- $d_{8}$ at room temperature. (C) DFT calculation of the ring-closure step in NCA synthesis with or without the assist of PO.

Several studies have demonstrated that the adverse effect of $\mathrm{HCl}$ can be minimized by employing an acid scavenger such as triethylamine (TEA), (+)-limonene, or $\alpha$-pinene, particularly in the synthesis of challenging NCAs.$^{38-40}$ We ruled out the use of TEA because i) it can prematurely initiate the ROP of NCA when in excess, ii) it cannot quench the nucleophilic chloride, which is known to attack the carbonyls of NCAs to form isocyanates, ${ }^{41}$ and iii) the removal of triethylammonium chloride salt is nontrivial. On the other hand, we tested $\alpha$-pinene but failed to improve the product yield or simplify the workup procedures over the conventional method (data not shown). Conducting the model reaction in a mixture of THF- $d_{8}, \alpha$-pinene $(1.0 \mathrm{M})$ and $\mathrm{HCl}(1.0$ $\mathrm{M})$ for $1 \mathrm{~h}$ at room temperature resulted in low conversion and slow rate (Figure 1B and S3). This is not surprising because $\alpha$-pinene has been reported to react with acid at a much slower rate in THF compared to in other solvents such as chloroform and xylene. ${ }^{42}$ Thus, a non-basic $\mathrm{HCl}$ scavenger 
with greater efficiency than $\alpha$-pinene was required.

We hypothesized that an epoxide such as propylene oxide (PO) and epichlorohydrin (ECH) could be ideal additives in NCA synthesis, due to their ability to remove both proton and chloride even at relatively low temperatures (Figure 1B) ${ }^{43}$ To our delight, the use of PO $(1.0 \mathrm{M})$ led to complete, rapid conversion of both reactants in less than $3 \mathrm{~min}$ (Figure 1B) at room temperature, generating a mixture of 1-chloro-2-propanol and 2-chloro-1-propanol (Figure S4). We thus anticipated that PO could greatly facilitate the synthesis of NCA at room temperature, presumably because the acid-catalyzed epoxide ring-opening could generate sufficient heat. Indeed, density functional theory (DFT) calculations confirmed that ethylene oxide could lower the energy barrier of the phosgenation reaction between $\mathrm{L}_{\mathrm{alanine}}$ and phosgene by $2.9 \mathrm{kcal} / \mathrm{mol}$ (Figure $1 \mathrm{C}$ and S5$6)$.

We then reasoned that the NCA synthesis could be performed under moisture because i) residual water in the solvent could be consumed by the excessive triphosgene, generating $\mathrm{CO}_{2}$ and $\mathrm{HCl}$, ii) all hydrogen chloride could be rapidly removed by PO (Scheme 1), and iii) PO, 1-chloro2-propanol, and 2-chloro-1-propanol are all volatile and easy to remove during the workup. To test the idea, we mixed $\gamma$-benzyl $\mathrm{L}$-glutamate ( $10 \mathrm{~g}, 1.0$ equiv), triphosgene ( 0.5 equiv), and PO (4.0 equiv) in regular THF $(150 \mathrm{~mL})$ at room temperature in a sealed $350-\mathrm{mL}$ heavy-wall flask. Unlike the conventional method, which would require heating the reaction mixture to $50-60{ }^{\circ} \mathrm{C}$ for $3-4 \mathrm{~h}$ under nitrogen, our new protocol achieved complete conversion of the amino acid substrate within $\sim 1.5 \mathrm{~h}$ without external heating. Thanks to the efficient and complete elimination of $\mathrm{HCl}$, pure BnGluNCA (Scheme 2, 3a) could be obtained at $84 \%$ yield following solvent removal and product recrystallization, under ambient conditions and without the need for anhydrous solvents or a glovebox (environmental humidity: 70\%). The high purity of Bn-GluNCA was confirmed by a combination of ${ }^{1} \mathrm{H}$ and ${ }^{13} \mathrm{C}$ NMR spectroscopy, high-resolution mass spectrometry, and FT-IR spectroscopy (Figure S7). Increasing the loading of PO from 4 to 10 equivalent showed little effect on the yield (See Table S1). The same protocol was then successfully applied to the preparation of ع-carboxybenzyl L-lysine NCA (Z-LysNCA, 3b), $\varepsilon$-trifluoroacetyl L-lysine NCA (TFA-LysNCA, 3c) L-analine NCA (AlaNCA, 3d), L-phenylalanine NCA (PheNCA, 3e), L-valine NCA (ValNCA, 3f), L-leucine NCA (LeuNCA, 3g), L-tryptophan NCA (TrpNCA, 3h), and L-tyrosine NCA (TyrNCA, 3i), all in satisfactory yields (64-90\%) after recrystallization (Scheme 2 and Figure S8-15). However, the yield for glycine NCA (GlyNCA) was below $50 \%$ even when the reaction was extended to $24 \mathrm{~h}$ (See the SI), likely due to the poor solubility of glycine in organic solvents. Surprisingly, complete glycine conversion was achieved within $13 \mathrm{~h}$ by simply replacing $\mathrm{PO}$ with $\mathrm{ECH}$, affording pure GlyNCA in 76\% yield after recrystallization (Scheme 2, 3j and Figure S16). While the exact reason of this phenomenon is still unknown, one hypothesis that is under investigation in our lab involves the cooperative hydrogen-halogen bond between glycine and the addition product of $\mathrm{ECH}$, which enhances the solubility of the substrate. Importantly, our method exhibited satisfactory compatibility with normal column chromatography for the synthesis of non-crystallizable NCAs. One notable example was the exceedingly hygroscopic $\gamma$-(2-(2-(2-methoxyethoxy)ethoxy)ethyl L-glutamate $\mathrm{NCA}\left(\mathrm{EG}_{3}-\mathrm{GluNCA}\right)$, which we were previously unable to synthesize at $40 \%$ environmental humidity and above, regardless of how other reaction conditions were optimized. In the current study, however, we successfully obtained $2.7 \mathrm{~g}$ of pure EG 3 -GluNCA (Scheme 2, 3k and Figure S17) with a separation yield of $72 \%$ by using regular solvents and flash column chromatography under air (environmental humidity: 66). The method also proved robust for the synthesis of ${ }_{\mathrm{L}}$-methionine 
NCA (MetNCA, Scheme 2, 31, Figure S18), $O$-diethylphospho L-tyrosine NCA (pOEt-TyrNCA, Scheme 2, 3m, Figure S19), O-tert-butyl L-serine NCA ( $t$ Bu-SerNCA, Scheme 2, 3n, Figure S20),

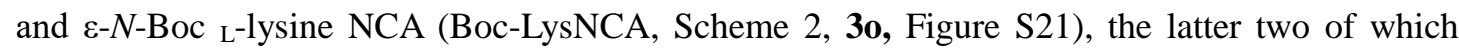
contained acid-labile protective groups that were difficult to handle previously. To fully illustrate the water-tolerance of the method, $\mathrm{H}_{2} \mathrm{O}$ (1 euqiv to amino acid) was intentionally added to the reaction mixture, and the synthesis of BnGluNCA still gave satisfactory results by slighlty increasing the loading of triphosegene (to 0.8 euqiv) and PO (to 6 equiv).

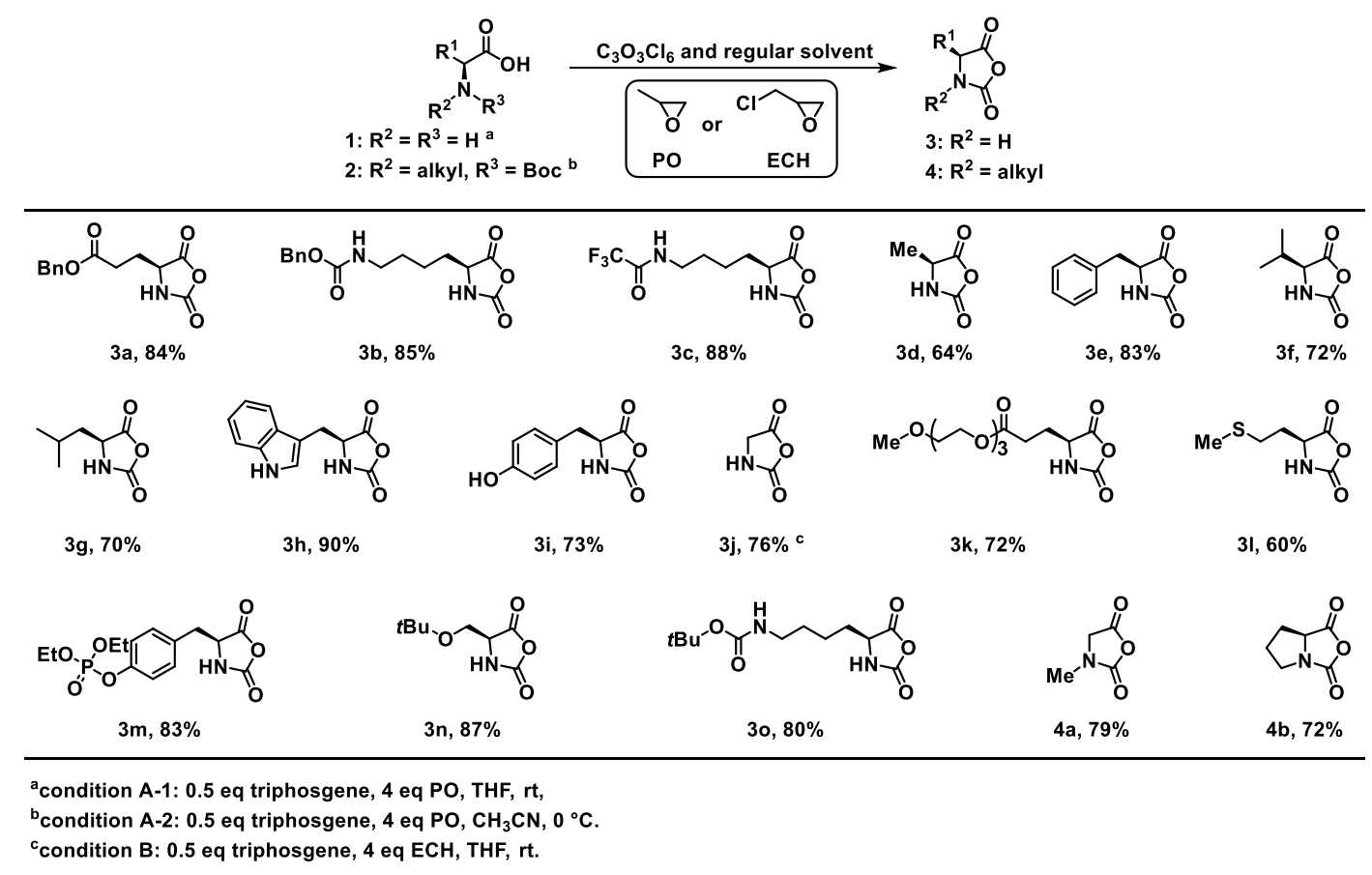

Scheme 2

Pivoting to $N$-substituted NCAs (NNCA), we surprisingly ran into roadblocks in our initial attempts to prepare sarcosine NCA (SarNCA) and ProNCA. Analysis of the crude reaction mixtures revealed the formation of by-products from the aminolysis of PO by the secondary amines (Figure S22). ${ }^{44}$ We thus employed $N$-Boc amino acids as starting materials, which had been previously applied to NCA synthesis with success. To our gratification, we isolated SarNCA in $79 \%$ yield by conducting the reaction in an open flask at $0^{\circ} \mathrm{C}$ to both ensure smooth gas release during the in-situ deprotection of Boc and prevent the loss of PO vapor (Scheme 2, 4a and Figure S23). The same strategy also proved successful in the synthesis of ProNCA (Scheme 2, 4b, and Figure S24) from $N$-Boc $\mathrm{L}$-proline, attaining a purified yield of $72 \%$ even at $74 \%$ environmental humidity.

To further showcase the functional group tolerance of the new method, we explored the generation of challenging NCAs that would otherwise be inaccessible. As reactive anhydrides, NCAs are thought to be incompatible with nucleophiles and/or reactive protons such as hydroxyl, amine, carboxylic acid and thiol. Consistently, the synthesis of hydroxyl-bearing NCAs, such as $\mathrm{L}^{-}$

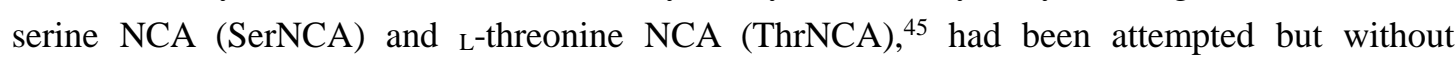
convincing characterization results. In contrast, we speculated that hydroxy-bearing NCAs should be well tolerated by our method, based on the fact that neither 1-chloro-2-propanol nor 2-chloro-1- 
propanol, the ring-opening products of $\mathrm{PO}$ and $\mathrm{HCl}$, exhibited any detrimental effect on product formation. Indeed, SerNCA (Scheme 3, 3p, and Figure S25) and ThrNCA (Scheme 3, 3q, and Figure S26) could be isolated at $71 \%$ and $46 \%$ yield (environmental humidity $68 \%$ ), respectively. The moderate decrease in the yield of ThrNCA was not due to low substrate conversion but rather the epimerization of the $\alpha$-carbon of threonine during the recrystallization, which led to diastereomers (Figure S27). As a further example, 4-hydroxy L-proline NCA (HypNCA, Scheme 3, 4c, and Figure S28) was also obtained in $75 \%$ yield by using $N$-Boc 4-hydroxy L-proline as the starting material. As a strong nucleophile, free thiol has not been directly introduced to the side group of NCA before, even though a few papers claimed the generation of such NCAs but without concrete characterization. ${ }^{46}$ To our great surprise, we successfully synthesized pure L-cysteine NCA (CysNCA, Scheme 3, 3r, Figure S29) bearing a primary thiol, and D-penicillamine NCA (PenNCA, Scheme 3, 3s, Figure S30) with a tertiary thiol. The production of the temperature-sensitive CysNCA was accomplished in $94 \%$ yield via recrystallization at $8-10{ }^{\circ} \mathrm{C}$ (See SI) and could be easily scaled up to $\sim 10 \mathrm{~g}$ per batch (see SI). The unprotected ${ }_{\mathrm{L}}$-glutamic acid NCA (GluNCA, Scheme 3, 3t, Figure S31) was also smoothly prepared with $\mathrm{ECH}$, but not with $\mathrm{PO}$, and $\sim 10$ grams of the product was obtained after recrystallization at a yield of $92 \%$. Again, it should be emphasized that all syntheses and workup procedures were conducted using regular solvents and without nitrogen protection, and none of these NCAs was found to be attainable using regular method or by adding $\alpha$-pinene as an additive ( $0 \%$ purification yield, Scheme 3 ). Corroborating the synthetic utility of unprotected NCAs, the ROP of GluNCA (3t) smoothly afforded poly-L-glutamic acid (PLG), a therapeutically relevant biodegradable carrier polymer being evaluated in phase 3 clinical trials, in one step with good molecular weight control (Figure S32-33). ${ }^{19}$ This concise synthesis avoided the

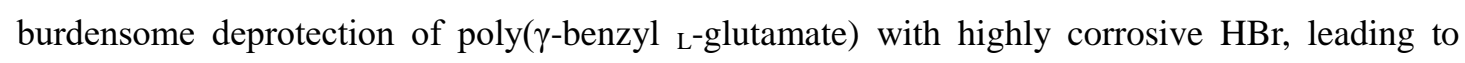
substantial time saving and yield increase compared to the conventional two-step procedure of ROP and deprotection ( $80 \%$ vs. $40 \%)$. 


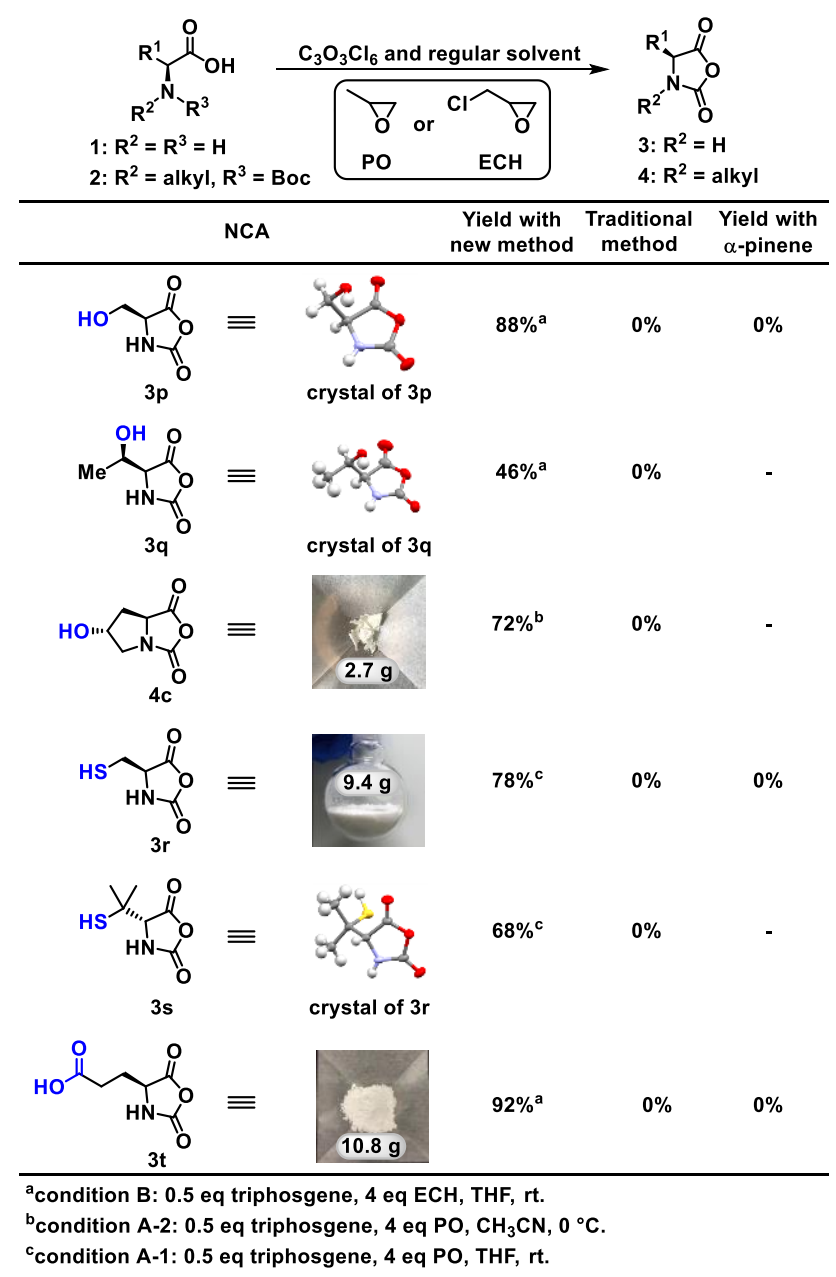

Scheme 3

Building on our success with NCAs, we set out to expand the scope of our new protocol to $\alpha$ hydroxyl acid $O$-carboxyanhydrides (OCA) $)^{47-49}$ and $\beta$-amino acid NCA ( $\left.\beta N C A\right) .{ }^{50}$ Similar to that of NCA, the synthesis of OCA generally involves stringent anhydrous conditions, but also requires activated charcoal to remove impurities. Here, by implementing PO as the acid scavenger, the representative L-phenyl lactic acid OCA (Scheme 4, 7a and Figure S34) and L-mandelic OCA (Scheme 4, 7b and Figure S35) were smoothly produced in modest yields (50-69\%). Of note, the synthesis of OCA required longer time than that of NCA for the reason that the former's hydroxy group is less nucleophilic than the latter's amino group. On the other hand, $\beta \mathrm{NCAs}$, which are considerably more labile than $\alpha$-amino acid NCAs, could also be conveniently synthesized in excellent yields (68-96\%; Scheme 4, 8a-f and Figure S36-41) with ECH as additive and under atmospheric conditions. Notably, $\beta$ NCAs prepared by the flow chemistry method were reported to result in easy product decomposition during the workup or storage. ${ }^{50}$ As a result, only H NMR yield, but not separation yield, were reported in the study on $\beta \mathrm{NCA}$ synthesis. Here, the enhanced stability of $\beta \mathrm{NCA}$ suggested an absence of side reactions due to the complete removal of $\mathrm{HCl}$, which once again underscored the power of our method. 


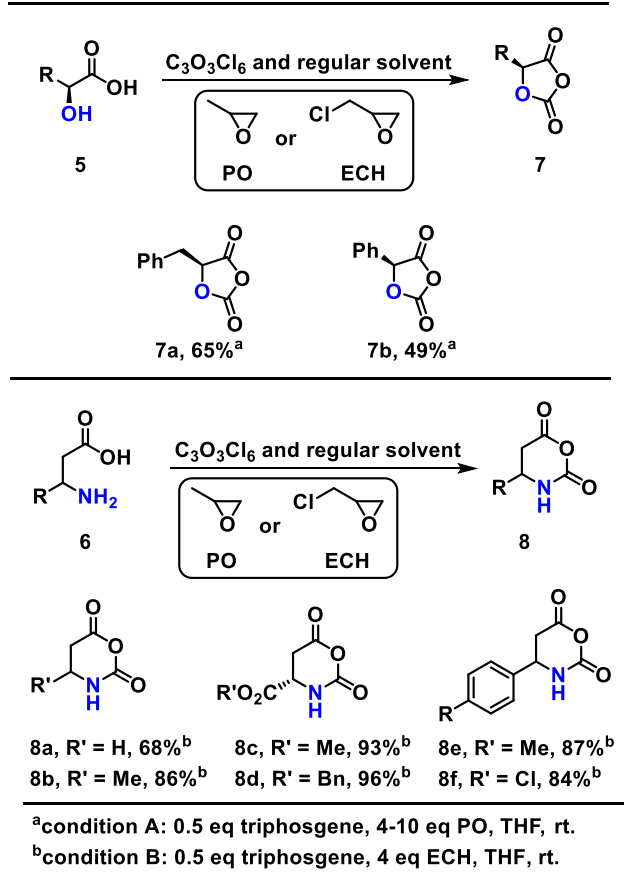

Scheme 4

Synthetic polypeptides have gained popularity as biodegradable and protein-mimicking polymers with broad application potential, but the preparation of the NCA monomers requires stringent moisture-free conditions and tricky workup procedures. Acid scavengers, such as TEA and $\alpha$-pinene, have been tested in an attempt to minimize the adverse effect of moisture and improve functional group compatibility, but with limited successes. Furthermore, the detrimental role of $\mathrm{HCl}$ in the formation of NCA, long recognized in the field, has not been elucidated from a mechanistic perspective until this work. After ruling out TEA and $\alpha$-pinene, we identified PO and ECH as ultrafast and clean $\mathrm{HCl}$-quenching reagents. We boldly proposed and later successfully proved, for the first time, that the above epoxides could be used to efficiently synthesize NCA under mild, atmospheric conditions, without the need for moisture removal. Subsequent investigations showed that our method could be generally applied to a broad scope of substrates, and could even be executed on a decagram scale to produce challenging NCAs that carry highly reactive - $\mathrm{OH}$, $-\mathrm{COOH}$, and -SH groups. The use of unprotected NCAs, such as HypNCA, SerNCA, and GluNCA, can undoubtedly improve the efficiency of polypeptide synthesis by obviating the need for tedious protection and deprotection. This is vividly demonstrated through the direct ROP of GluNCA (3t). Moreover, CysNCA could be treated as an inimer, allowing one-pot production of hyperbranched polypeptides. The generality of the method was further demonstrated by the synthesis of several OCAs and $\beta$ NCAs in high purity, paving the road for easier access to various functional poly $(\alpha-$ hydroxyl acid)s and $\beta$-polypeptides. These works will be reported in separated papers shortly. Last but not least, we expect the method detailed in this study to be readily adaptable to industrial-scale production for reasons that i) both $\mathrm{PO}$ and $\mathrm{ECH}$ are extremely inexpensive and easily available, ii) the reactions can be performed without the need for ultra-dry solvents, nitrogen protection or glovebox, leading to much simpler procedures and considerable cost reduction; iii) the instant removal of $\mathrm{HCl}$ can prevent the erosion of equipment, and iv) the solvents, $\mathrm{PO}, \mathrm{ECH}$, and the ringopening byproducts can all be easily recycled through distillation. Taken together, the simplicity, 
robustness and scalability of our method promises may ultimately revolutionize the NCA synthesis, profoundly facilitate the popularization of synthetic polypeptides in both laboratories and industry, and open up numerous possibilities for the development of novel, high-performance polypeptide materials.

\section{Supporting Information}

Experimental details, ${ }^{1} \mathrm{H}$ and ${ }^{13} \mathrm{C}$ NMR spectra, kinetic studies, DFT calculations.

X-ray crystallographic data (3p)

X-ray crystallographic data $(3 q)$

X-ray crystallographic data (3r)

\section{Notes}

A provisional patent application has been filed by H.L. and Z.Y.T.

\section{Corresponding Author}

*chemhualu@pku.edu.cn.

\section{Acknowledgment}

This work is supported by the National Natural Science Foundation of China (21722401 and 21975004) and Li Ge-Zhao Ning Life Science Research Foundation for Young Scholars. Z. Y. T. was supported by the National Postdoctoral Program for Innovative Talents of China (BX20190004). The computation was supported by High-performance Computing Platform of Peking University. The authors thank Mr. Shuo Wang for his help on the recrystallization of CysNCA.

\section{References}

1. Rasines Mazo, A.; Allison-Logan, S.; Karimi, F.; Chan, N. J.; Qiu, W.; Duan, W.; O'BrienSimpson, N. M.; Qiao, G. G., Ring opening polymerization of alpha-amino acids: advances in synthesis, architecture and applications of polypeptides and their hybrids. Chem. Soc. Rev. 2020, 49 (14), 4737-4834.

2. Shen, Y.; Fu, X.; Fu, W.; Li, Z., Biodegradable stimuli-responsive polypeptide materials prepared by ring opening polymerization. Chem. Soc. Rev. 2015, 44 (3), 612-22.

3. Hadjichristidis, N.; Iatrou, H.; Pitsikalis, M.; Sakellariou, G., Synthesis of Well-Defined Polypeptide-Based Materials via the Ring-Opening Polymerization of alpha-Amino Acid NCarboxyanhydrides. Chem. Rev. 2009, 109 (11), 5528-5578.

4. Klok, H. A.; Lecommandoux, S., Solid-state structure, organization and properties of peptidesynthetic hybrid block copolymers. Adv. Polym. Sci. 2006, 202, 75-111.

5. Hou, Y.; Zhou, Y.; Wang, H.; Sun, J.; Wang, R.; Sheng, K.; Yuan, J.; Hu, Y.; Chao, Y.; Liu, Z.; Lu, H., Therapeutic Protein PEPylation: The Helix of Nonfouling Synthetic Polypeptides Minimizes Antidrug Antibody Generation. ACS Cent. Sci. 2019, 5 (2), 229-236.

6. Hou, Y.; Lu, H., Protein PEPylation: A New Paradigm of Protein-Polymer Conjugation. Bioconjugate Chem. 2019, 30 (6), 1604-1616.

7. Zagorodko, O.; Arroyo-Crespo, J. J.; Nebot, V. J.; Vicent, M. J., Polypeptide-Based Conjugates as Therapeutics: Opportunities and Challenges. Macromol. Biosci. 2017, 17 (1), 1600316.

8. Cabral, H.; Kataoka, K., Progress of drug-loaded polymeric micelles into clinical studies. $J$. 
Controlled Release 2014, 190, 465-476.

9. Anderson, M. A.; O'Shea, T. M.; Burda, J. E.; Ao, Y.; Barlatey, S. L.; Bernstein, A. M.; Kim, J. H.; James, N. D.; Rogers, A.; Kato, B.; Wollenberg, A. L.; Kawaguchi, R.; Coppola, G.; Wang, C.; Deming, T. J.; He, Z. G.; Courtine, G.; Sofroniew, M. V., Required growth facilitators propel axon regeneration across complete spinal cord injury. Nature 2018, 561 (7723), 396-400.

10. Sun, Y. T.; Wollenberg, A. L.; O'Shea, T. M.; Cui, Y. X.; Zhou, Z. H.; Sofroniew, M. V.; Deming, T. J., Conformation-Directed Formation of Self-Healing Diblock Copolypeptide Hydrogels via Polyion Complexation. J. Am. Chem. Soc. 2017, 139 (42), 15114-15121.

11. Xiong, M.; Lee, M. W.; Mansbach, R. A.; Song, Z.; Bao, Y.; Peek, R. M.; Yao, C.; Chen, L. F.; Ferguson, A. L.; Wong, G. C. L.; Cheng, J. J., Helical antimicrobial polypeptides with radial amphiphilicity. Proc. Natl. Acad. Sci. U. S. A. 2015, 112 (43), 13155-13160.

12. Lam, S. J.; O'Brien-Simpson, N. M.; Pantarat, N.; Sulistio, A.; Wong, E. H. H.; Chen, Y. Y.; Lenzo, J. C.; Holden, J. A.; Blencowe, A.; Reynolds, E. C.; Qiao, G. G., Combating multidrugresistant Gram-negative bacteria with structurally nanoengineered antimicrobial peptide polymers. Nat. Microbiol. 2016, 1 (11), 16162.

13. Hanson, J. A.; Chang, C. B.; Graves, S. M.; Li, Z. B.; Mason, T. G.; Deming, T. J., Nanoscale double emulsions stabilized by single-component block copolypeptides. Nature 2008, 455 (7209), 85-88.

14. Song, Z.; Han, Z.; Lv, S.; Chen, C.; Chen, L.; Yin, L.; Cheng, J., Synthetic polypeptides: from polymer design to supramolecular assembly and biomedical application. Chem. Soc. Rev. 2017, 46 (21), 6570-6599.

15. Manai, G.; Houimel, H.; Rigoulet, M.; Gillet, A.; Fazzini, P.-F.; Ibarra, A.; Balor, S.; Roblin, P.; Esvan, J.; Coppel, Y.; Chaudret, B.; Bonduelle, C.; Tricard, S., Bidimensional lamellar assembly by coordination of peptidic homopolymers to platinum nanoparticles. Nat. Commun. 2020, 11 (1), 2051. 16. Baars, S.; Drauz, K. H.; Krimmer, H. P.; Roberts, S. M.; Sander, J.; Skidmore, J.; Zanardi, G., Development of the Julia-Colonna asymmetric epoxidation reaction: Part 1. Preparation and activation of the polyleucine catalyst. Org. Process Res. Dev. 2003, 7 (4), 509-513.

17. Racke, M. K.; Lovett-Racke, A. E.; Karandikar, N. J., The mechanism of action of glatiramer acetate treatment in multiple sclerosis. Neurology 2010, 74 (1), S25-S30.

18. Song, Z. Y.; Khaw, Y. M.; Pacheco, L. A.; Tseng, K. Y.; Tan, Z. Z.; Cai, K. M.; Ponnusamy, E.; Cheng, J. J.; Inoue, M., Induction of a higher-ordered architecture in glatiramer acetate improves its biological efficiency in an animal model of multiple sclerosis. Biomater. Sci. 2020, 8 (19), 52715281.

19. Zhao, J.; Koay, E. J.; Li, T.; Wen, X.; Li, C., A hindsight reflection on the clinical studies of poly(l-glutamic acid)-paclitaxel. Wiley Interdiscip. Rev. Nanomed. Nanobiotechnol. 2018, 10 (3), e1497.

20. Zhao, W.; Lv, Y.; Li, J.; Feng, Z.; Ni, Y.; Hadjichristidis, N., Fast and selective organocatalytic ring-opening polymerization by fluorinated alcohol without a cocatalyst. Nat. Commun. 2019, 10 (1), 3590 .

21. Grazon, C.; Salas-Ambrosio, P.; Ibarboure, E.; Buol, A.; Garanger, E.; Grinstaff, M. W.; Lecommandoux, S.; Bonduelle, C., Aqueous Ring-Opening Polymerization-Induced SelfAssembly (ROPISA) of N-Carboxyanhydrides. Angew. Chem., Int. Ed. 2020, 59 (2), 622-626.

22. Song, Z.; Fu, H.; Wang, J.; Hui, J.; Xue, T.; Pacheco, L. A.; Yan, H.; Baumgartner, R.; Wang, Z.; Xia, Y.; Wang, X.; Yin, L.; Chen, C.; Rodríguez-López, J.; Ferguson, A. L.; Lin, Y.; Cheng, J., 
Synthesis of polypeptides via bioinspired polymerization of in situ purified $\mathrm{N}$-carboxyanhydrides. Proc. Natl. Acad. Sci. U. S. A. 2019, 116 (22), 10658-10663.

23. Wu, Y.; Zhang, D.; Ma, P.; Zhou, R.; Hua, L.; Liu, R., Lithium hexamethyldisilazide initiated superfast ring opening polymerization of alpha-amino acid N-carboxyanhydrides. Nat. Commun. 2018, 9 (1), 5297.

24. Yuan, J.; Zhang, Y.; Li, Z.; Wang, Y.; Lu, H., A S-Sn Lewis Pair-Mediated Ring-Opening Polymerization of $\alpha$-Amino Acid N-Carboxyanhydrides: Fast Kinetics, High Molecular Weight, and Facile Bioconjugation. ACS Macro Lett. 2018, 7 (8), 892-897.

25. Yuan, J.; Sun, Y.; Wang, J.; Lu, H., Phenyl Trimethylsilyl Sulfide-Mediated Controlled RingOpening Polymerization of alpha-Amino Acid N-Carboxyanhydrides. Biomacromolecules 2016, 17 (3), 891-896.

26. Jacobs, J.; Pavlović, D.; Prydderch, H.; Moradi, M.-A.; Ibarboure, E.; Heuts, J. P. A.; Lecommandoux, S.; Heise, A., Polypeptide Nano particles Obtained from Emulsion Polymerization of Amino Acid N-Carboxyanhydrides. J. Am. Chem. Soc. 2019, 141 (32), 12522-12526.

27. Cao, J. B.; Siefker, D.; Chan, B. A.; Yu, T. Y.; Lu, L.; Saputra, M. A.; Fronczek, F. R.; Xie, W. W.; Zhang, D. H., Interfacial Ring-Opening Polymerization of Amino-Acid-Derived NThiocarboxyanhydrides Toward Well-Defined Polypeptides. ACS Macro Lett. 2017, 6 (8), 836-840. 28. Tao, X. F.; Li, M. H.; Ling, J., alpha-Amino acid N-thiocarboxyanhydrides: A novel synthetic approach toward poly(alpha-amino acid)s. Eur. Polym. J. 2018, 109, $26-42$.

29. Conejos-Sanchez, I.; Duro-Castano, A.; Birke, A.; Barz, M.; Vicent, M. J., A controlled and versatile NCA polymerization method for the synthesis of polypeptides. Polym. Chem. 2013, 4 (11), 3182-3186.

30. Zou, J.; Fan, J. W.; He, X.; Zhang, S. Y.; Wang, H.; Wooley, K. L., A Facile Glovebox-Free Strategy To Significantly Accelerate the Syntheses of Well-Defined Polypeptides by NCarboxyanhydride (NCA) Ring-Opening Polymerizations. Macromolecules 2013, 46 (10), 42234226.

31. Huesmann, D.; Klinker, K.; Barz, M., Orthogonally reactive amino acids and end groups in NCA polymerization. Polym. Chem. 2017, 8 (6), 957-971.

32. Kricheldorf, H. R., Polypeptides and 100 years of chemistry of alpha-amino acid Ncarboxyanhydrides. Angew. Chem., Int. Ed. 2006, 45 (35), 5752-5784.

33. Deming, T. J., Synthesis of Side-Chain Modified Polypeptides. Chem. Rev. 2016, 116 (3), 786808.

34. Song, Z. Y.; Tan, Z. Z.; Cheng, J. J., Recent Advances and Future Perspectives of Synthetic Polypeptides from N-Carboxyanhydrides. Macromolecules 2019, 52 (22), 8521-8539.

35. Wu, G. Q.; Ge, C. L.; Liu, X. Y.; Wang, S.; Wang, L. T.; Yin, L. C.; Lu, H., Synthesis of water soluble and multi-responsive selenopolypeptides via ring-opening polymerization of $\mathrm{N}$ carboxyanhydrides. Chem. Coтmum. 2019, 55 (54), 7860-7863.

36. Kramer, J. R.; Deming, T. J., General Method for Purification of alpha-Amino acid-Ncarboxyanhydrides Using Flash Chromatography. Biomacromolecules 2010, 11 (12), 3668-3672.

37. Otake, Y.; Nakamura, H.; Fuse, S., Rapid and Mild Synthesis of Amino Acid N-Carboxy Anhydrides: Basic-to-Acidic Flash Switching in a Microflow Reactor. Angew. Chem., Int. Ed. 2018, 57 (35), 11389-11393.

38. Fabrice Cornille, B. S. Y.; Jean-Luc Copier, A.; Jean-Pierre Senet, B.; Yves Robin, V. L. P. PROCESS FOR THE PREPARATION OF N-CARBOXYANHYDRIDES. US 6,479,665 B2, 2002. 
39. Iizuka, Y.; Uchida, C.; Wakamatsu, K.; Oya, M., Synthesis and Properties of High-MolecularWeight Polypeptides Containing Proline. Bull. Chem. Soc. Jpn. 1993, 66 (4), 1269-1272.

40. Smeets, N. M. B.; van der Weide, P. L. J.; Meuldijk, J.; Vekemans, J. A. J. M.; Hulshof, L. A., A scalable synthesis of L-leucine-N-carboxyanhydride. Org. Process Res. Dev. 2005, 9 (6), 757-763.

41. Iwakura, Y.; Uno, K.; Kang, S., The Synthesis and Reactions of 2-Isocyanatoacyl Chlorides. $J$. Org. Chem. 1965, 30 (4), 1158-1161.

42. Hennion, G. F.; Irwin, C. F., Solvent Effects in Addition Reactions. II. Addition of Hydrogen Bromide and Chloride to $\alpha$-Pinene. J. Am. Chem. Soc. 1941, 63 (3), 860-862.

43. Stewart, C. A.; Vanderwerf, C. A., Reaction of Propylene Oxide with Hydrogen Halides. J. Am. Chem. Soc. 1954, 76 (5), 1259-1264.

44. Lehmann, J., Lactone, 2. Mitt.1) Synthese dihydroxylierter Diphenylalkylamine über Azalactone. Archiv. der Pharmazie. 1983, 316 (4), 339-346.

45. Saito, T., A New Rearrangement Reaction of Beta-Hydroxy-Alpha-Amino Acid N-Carboxy Anhydrides to 2-Oxazolidone Derivatives. Bull. Chem. Soc. Jpn. 1964, 37 (5), 624-628.

46. Hirschmann, R.; Schwam, H.; Strachan, R. G.; Schoenewaldt, E. F.; Barkemeyer, H.; Miller, S. M.; Conn, J. B.; Garsky, V.; Veber, D. F.; Denkewalter, R. G., Controlled synthesis of peptides in aqueous medium. VIII. Preparation and use of novel alpha.-amino acid N-carboxyanhydrides. $J$. Am. Chem. Soc. 1971, 93 (11), 2746-2754.

47. du Boullay, O. T.; Marchal, E.; Martin-Vaca, B.; Cossio, F. P.; Bourissou, D., An activated equivalent of lactide toward organocatalytic ring-opening polymerization. J. Am. Chem. Soc. 2006, 128 (51), 16442-16443.

48. Wang, R. B.; Zhang, J. W.; Yin, Q.; Xu, Y. X.; Cheng, J. J.; Tong, R., Controlled Ring-Opening Polymerization of O-Carboxyanhydrides Using a beta-Diiminate Zinc Catalyst. Angew. Chem., Int. Ed. 2016, 55 (42), 13010-13014.

49. Li, M. S.; Tao, Y.; Tang, J. D.; Wang, Y. C.; Zhang, X. Y.; Tao, Y. H.; Wang, X. H., Synergetic Organocatalysis for Eliminating Epimerization in Ring-Opening Polymerizations Enables Synthesis of Stereoregular Isotactic Polyester. J. Am. Chem. Soc. 2019, 141 (1), 281-289.

50. Sugisawa, N.; Otake, Y.; Nakamura, H.; Fuse, S., Single-Step, Rapid, and Mild Synthesis of beta-Amino Acid N-Carboxy Anhydrides Using Micro-Flow Technology. Chem-Asian J. 2020, 15 (1), 79-84. 
